\title{
Design and Application of Remote-Learner's Affective Testing in Human- Computer Interaction
}

\author{
Gui-Feng $\mathrm{Wu}^{1,2, *}$, Sheng-Quan $\mathrm{Li}^{1}$ and Hong-Ru Tang ${ }^{1}$ \\ ${ }^{I}$ Department of Electrical Engineering, Yangzhou University, Yangzhou 225127, China; ${ }^{2}$ Institute of Psychology, \\ Chinese Academy of Sciences, Beijing 100101, China
}

\begin{abstract}
Based on education agent application, the paper designed emotional detection model and overall schema of detection for learners. Then the paper completed personality learning monitoring for learners and defined emotional attitude, cognitive state and learning preference for learners through extraction and analysis on emotional information between education agent and learners. The paper conducted personalized learning resources service for learners by using automatically generated courses tool based on emotional detection results in order to improve human-computer interaction status in remote learning and improve overall learning effect. The paper also studied on evaluation method of human-computer interaction system and focused on the multi-objective decision-making method. Then the paper realized more accurate quantitative evaluation through evaluation of NAP on human-computer interaction.
\end{abstract}

Keywords: Affective testing, design and application, human-computer interaction, remote-learner.

\section{INTRODUCTION}

Distance education by its flexibility, convenience, and sharing of, for many normal school to participate in the study population provides continuing education opportunities, saving the cost of personnel training, expanding the training teams, for the enhancement of the quality of the people plays an important role. In recent years, as the "service" concept was introduced to distance education, distance education research of perspective gradually slopes towards the object of our service-learners. "Support service" is proposed under this concept, and more and more distance education researcher's attention. However, due to the separation of distance education students, resulting in emotional communication than in the traditional education is more difficult. Often distance learners responses are basically alone computer to complete the learning activities and therefore often feel alone in learning. Practice also shows that years of distance education distance education actual effect and high expectations held by people form a sharp contrast: learners' learning adaptability of distance education is not strong, weak selfcontrol, anxiety and loneliness of growing, poor learning and so on. These realities reveals lack of emotional issues in distance learning but also emotional demands of distance learners $[1,2]$.

A computer system is composed of computer hardware, software and people together constitute the human-machine system. Human-computer interaction is on the design, evaluation and implementation of interactive computer system for people to use and around these main scientific phenomenons to study.
Human-computer interaction and cognitive psychology, ergonomics, multimedia and virtual reality are closely related. Human-machine interface is people interacting with the machine's operation, namely the user medium for transmitting information to each other with machines. Humanmachine interface and interaction are two different concepts, refers to human-computer interaction between man and computer symbols and two-way exchange of information and the human-machine interface is man-machine communication media and tools which is a two-way exchange of information to support software and hardware. But between the human-machine dialogue is realized by certain humanmachine interface, so the two are closely linked $[3,4]$.

Affective Computing was proposed from the United States Picard in 1995. In the book she defines "affective computing is emotion-related, from emotions or to influence the emotions of calculation". It is called affective computing trying to make computers as human observation, understanding and the ability to generate a variety of emotions [5]. Affective computing research is an attempt to create a perception, recognition and understanding of human emotions and human emotions to make intelligent, sensitive and friendly response calculation system. Study on Picard affective computing content divided into nine areas: access to mechanism of emotion, emotion, emotional pattern recognition, modelling and understanding of emotion, emotion synthesis, emotional expression, affective computing, application of computer interface, the transfer and exchange of emotion, wearable computer. Current work focuses on the emotional signal acquisition and identification. Emotional calculation can from two a aspects understanding: $a$ is based on physiology of angle, through various measurement means detection human of various physiological parameter, as heartbeat, and pulse, and brain radio, and to this for according to calculation human of emotional state; the second is based on psy- 
chology of angle, through various sensor accept and processing environment information, and to this for according to calculation artificial machine (as personal robot) of emotional state $[6,7]$.

Next, we describe the data and methodology used in more detail.

\section{PRINCIPLES AND METHODS}

Human-computer interaction research is very extensive, covering modelling, design, evaluation theory and methods, as well as on the Web, mobile computing, virtual reality applications in areas such as research and development, include the following: 1) Interface model and its design method. Advantages and disadvantages of an interactive interface, directly affects the success of software development. Friendly user interface development is inseparable from good interaction model and design methods. Therefore, the interface model and design methods are an important part of humancomputer interaction. 2) Availability of analysis and evaluation. Usability is an important part of the human-computer interaction system, which related to human-computer interaction can achieve the goal of users expect, and the achievement of the goals of efficiency and convenience. On humanmachine interaction system availability analysis and evaluation research comes to support the available design principles and usability evaluation methods. 3) Multimodal interaction techniques. In multimodal interaction, users can use the voice, gestures, eye contact, facial expression, natural way to communicate with the computer system. Multimodal interaction studies of multimodal interface model, multichannel interface evaluation methods of multi-channel information fusion, etc. Among them, multimodal integration is the focus of multimodal user interfaces and difficulty. 4) Cognition and intelligent user interface. Intelligent user interfaces' ultimate goal is to enable human-machine interaction as natural and human-human interaction and convenience. Context-awareness, eye-tracking, gesture recognition, three dimensional input, speech recognition, facial recognition, handwriting recognition, natural language understanding, are recognition and intelligent user interfaces need to address the important issues. 5) Virtual environment interaction. "People-oriented", natural harmony of human-computer interaction theory and methods is one of the main contents of virtual reality. Through research and Visual, auditory, tactile and other channels of information fusion theory, collaboration technologies and methods, as well as the three-way, creating highly realistic virtual environment, to create "immersive" feeling. 6) Mobile interface design. Mobile. Computing, relevant general computing, human-machine interaction technology put forward higher requirements, interface design for mobile applications have become an important application field of human-machine interaction technology research. Due to the portability of mobile devices, location is not fixed, limited computing power and unlimited restrictions on low bandwidth, high latency, and many other networks, mobile interface, mobile interface usability and evaluation principles, mobile interface navigation techniques and realization of mobile interfaces and development tools, is the current hot spot in human-machine interaction technology. 7) Group. Groupware is defined as a group that work together to provide computer support for collaborative envi- ronment, mainly related to personal or group messaging, group information sharing, business process automation and coordination and interaction between people and process activities. Now and man-machine interaction technologyrelated research includes groupware system architecture, computer supported communication and sharing of information, exchange of decision support tools, application sharing, and synchronization methods.

Typically, people use eye, ear, nose, tongue, and skin as a sensor, communication with the outside world. In emotional communication, information that is issued by the five sense organs for emotional information, including emotional information, visual hearing emotion emotional information and emotional information, smell, taste, tactile emotion. (1) Visual emotion. Human vision is the main source of information, but also people understanding and evaluation of window on the world. From a person's face showed sadness, anger, surprise, fear, and happy expression can understand the heart of happiness, anger, sadness and joy, mental state, the expression can be said to be a kind of Visual emotion. (2) Hearing emotional information. Music is the essence of auditory information, music as emotional, turbulent moments, there are moments of calm, violent, and quiet. Note has no meaning, but it can evoke fear, anger, surprise, uncertainty, frustration, calm and love and emotion. (3) Smell, emotion. Smell is quiet consciousness, wordless sense with every breath, always smell. Fragrance, scent, sandalwood and the stench is the smell of emotional information. (4) Taste, emotional information. Emotional information is so-called taste, sour, sweet, bitter, hot and salty. (5) Visual emotion. Touch is one of the oldest and most important sense. Smooth, rough, cold, soft and hard, and so is the touch of emotion.

In this study, the emotional information refers to the learners in the learning process, as the study progresses, appear happy, sad, anger, surprise, disgust, and to accept emotions. It's mainly for the eyes, lips, eyebrows, nose and other facial expressions and verbal expression and body changes. Emotion detection model is divided into four key steps, among them, the first step is the feeling for human-computer interaction in the process of information gathering and extraction; The second step is to collect and extract the emotion and emotion mapping model; Third step is in on mapping results analysis completed on learning who overall learning monitoring results of data analysis, to precise master learning who of personality learning features and cognitive State and learning preference,, to for learning who provides further of learning service; fourth step is through automatically generated courses tool completed courses of personalized design, for learning who provides effective and personalized of courses resources worth note of is, whole technology process is constantly cycle of, To keep accurate on the learner's emotion detection and effective feedback learning resources. Which correspond to the four key technology modules for human-computer interaction process modules, respectively, affective model module, monitoring module, learning resource services module. Module is responsible for the process of human-computer interaction with learners in the learning process as a "Mentor" emotional exchange education agent are responsible not only for voice and text interaction with learners, and individualized learning content to the learner, complete the transit delivery processes for user ses- 
sion information and knowledge objects. Affective model module for fine resolution is responsible for collecting information about emotion, and emotion with the emotion model mapping after semantic annotation of emotion and emotion corpus matching meta-data in to determine the learner's emotional state at different levels in the learning process.

Learning module responsible for mastering the emotions of the learners in the learning process, emotional attitude to different learning content, personalized learning monitoring information for storage and analysis, which was further informed that the cognitive and learning preferences, and constantly improve the accuracy of monitoring information for learners, provides more effective learning services for learners. Learning resources service module is responsible for on emotional detection results be using, to this to for learning who provides effective, appropriate, personalized of learning feedback, makes whole remote learning process more effective, makes learning who can effective feel to knowledge, skills level of improve, meet learning who on learning effect of expected, while can in learning process in the has moments was concern of emotional experience, meet learning who of emotional hopes, avoid produced alone, helpless of learning mood. Human communication is formed by the combination of verbal communication and non-verbal communication. Two forms of communication including text and voice and non-verbal communication includes two forms of facial expressions and body movements. Emotional transmission process is the process of human communication, emotional transfer contained in human interaction. Expression of emotion through the four main forms: text, voice, facial expressions and body movements. Expressions (or external embodiment) perspective, it can be divided into language and the tone expression (including text and voice), facial and body expressions.

Language voice expression: the use of language and text (that is, the meaning of the word) this kind of abstract symbols to enable humans to more abstract, indirect and widespread awareness of the value of things and react. In addition, languages also can rely on speed, change of tone, sound intensity to determine and adjust its emotional form, so that the value of language can express more detailed content. As brisk tone may reflect a person's feeling of joy, deep tone, slow pacing may reflect a mood of depression and so on. Language can express complex feelings, if coupled with appropriate tones (such as strength, speed of sound, rhythm, etc.), can be rich, vivid and complete and accurately express one's emotional state, show the cultural level of the people, values and character traits. Facial expression: as the face closest to the brain, can quickly and accurately express your feelings, and features all together and people through the eyes, eyebrows, mouth, nose, facial muscles and other changes in different combinations to flexible, timely, accurate and detailed expression of his feelings. As joy when eyebrows stretch, smile; worried brows when locked, her lips tight are through facial expressions convey emotions such as SIP. Body expression: people's emotional state, capabilities and personality traits sometimes by gestures of the body spontaneously or consciously express to form the body expression. When a person is in a strong sense of excitement, tension, fear, anger, and other emotional States, often gave in to body expression, actors are often exaggerated body gestures to consciously express role of emotions. Human body conveyed is rich and diverse. Sat their respectful or nervous, danced joy, wringing its annoying.

In the distance learning environment, students and teachers separated in different places. Enrich the interaction between people and tools at the same time, emotional expression is increasingly in the form of a single. Includes associated with remote-learner interaction between teachers and students, between students, student interaction with the service. Emotions triggered by certain stimuli, remote learning, learners' experiences and the stimulus has its particularity, is mainly produced by learners in the learning process of emotional experience. Compared with general human emotions, distance learning, academic emotion still require further exploration and refining. By Perkin and others the concept of academic emotions was first proposed in 2002. They think academic emotions and learner motivation, self-closely related to the concept of relationship. Wide range of the emotions, refers to the process of teaching or learning, various academic and emotional experiences, such as joy, disappointment, anxiety, boredom, and anger and so are emotions. Academic emotions as compared to human emotions, there are three obvious characteristics: (1) academic emotions have diversity. Learners in the learning process, can produce a variety of emotions. (2) Academic emotions situated. In different learning contexts learners will have a different mood. Academic emotions will be learning interaction, learning requirements and environmental impact. (3) Academic emotions are dynamic. For the same learner, academic emotion is not always the same, but will change with the change of learning tasks and learning situations.

At present there are five main ways of emotional measurement: the self-report, interview, observation, projection and neural measurements. (1) Self-report: subjects were asked to rank scale or the adjective form to express your emotion through self-reporting to obtain the quantitative information of the learners are experiencing. (2) Interviews: it is through face-to-face conversation with learners, to learn about the learner's emotional state. (3) Method of observation: is the observer of "ongoing" behaviour were observed, and is usually observed for records. (4) Projection: also known as projective tests, psychological explanation, referring to his thoughts, attitudes, desires, emotions, or features, such as, unconsciously, a psychological reaction to external things or others. Due to the projected test takes too long and the operator's skills requirements are very high, so little on actual measurement of emotions. (5) The measurement of nerve: neurophysiological measurements are from the perspective of neuroscience, through the central nervous system and peripheral nervous system interact to understand the mechanism of emotion processing.

Self-reported law applies in practice the two requirements: (1) on the subjects of cognitive evaluation capacities have certain requirements. Such as when distance learners ' emotional state, learners must understand the meaning of various emotional variables, can better judge their emotional categories. (2) Has certain requirements for test time. Selfreport rating scale is required in the form of test subjects. Common forms are: three, five, seven and nine in several 
Table 1. A five-point scale.

\begin{tabular}{|c|c|c|c|c|c|}
\hline \multirow{2}{*}{ Emotional variables } & None & Minor & Moderate & Strong & Extreme \\
\cline { 2 - 6 } & 1 & 2 & 3 & 4 & 5 \\
\hline
\end{tabular}

forms. Too few three-point levels do not reflect well the learner's emotional level, and seven points and nine points is too much, too many subdivisions, are difficult to differentiate, not very meaningful. This research study will adopt a five-point scale; the learner's emotional level is divided into five levels, each with a Word to describe. If no record 1 minute, slightly to 2 points, recorded as 3 minutes, relatively strong for 4 points, quite strongly for 5 minutes.

Evaluation indicators can be divided into two types of quantitative and qualitative indicators. Quantitative indicators can be calculated according to the information available; for quantitative analysis but it is difficult to accurately calculate the indicators or qualitative indicators, set-valued statistic method is used.

$x(u)=\frac{1}{n} \sum_{k=1}^{n} x\left[u_{1}^{(\mathrm{k})}, u_{2}^{(\mathrm{k})}\right]^{(u)}$

Remote learning process in the on learning who and education agent of detection system and feedback mechanism of dialogue interactive, General for dialogue partners (learning who and education agent) of short voice and short text Exchange $\$$ which, on voice interactive signal of collection, is on Sonic direction, rhythm, base frequency and speed for extraction, will voice signal transcription for text information and for emotional detection of technology process. In this a detection steps in the, education agent main is completed and learning whose dynamic interactive and feedback information of dynamic rendering, as "Mentor" of education agent not only for learning who provides learning guide, and emotional exchange, while also is responsible for learning situation of created, due to learning who by learn subject and courses content the not same, while and are has itself of subject features and relative fixed of situation space, so, for effective traction learning who learning State, makes its input to learning situation in the, corresponding of situation support is necessary, Visual model and education agents were able to provide cognitive shortcuts for the learners ' thinking. But this feature is not overwhelmingly occupied the learner's thinking, is more concerned about the interaction between them, designed according to the characteristics, roles such as plays, films, comics character, habits, manners, opinions, designs, to attract the attention of learners and guide learners complete the course content.

$$
\begin{aligned}
& X=U * \sum * V^{T} \\
& X^{\prime}=\sum \circ-1 * U^{T} * X
\end{aligned}
$$

Evaluation index of dimensionless uses gravy system theory of effect measure.

For the indicator which became better and bigger:

$$
z(i, j)=S_{\min }+\frac{e(i, j)-\min _{j} \mathrm{e}(i, j)}{\max _{j} \mathrm{e}(i, j)-\min _{j} \mathrm{e}(i, j)} \times\left(S_{\max }-S_{\min }\right)
$$

For the indicator which became better and smaller:

$$
z(i, j)=S_{\max }-\frac{e(i, j)-\min _{j} \mathrm{e}(i, j)}{\max _{j} \mathrm{e}(i, j)-\min _{j} \mathrm{e}(i, j)} \times\left(S_{\max }-S_{\min }\right)
$$

For e-plus in some appropriate value indicators:

$z(i, j)=S_{\max }-\frac{\left|e(i, j)-e_{0}\right|}{\left|e(i, j)-e_{0}\right|+\mathrm{e}_{0}} \times\left(S_{\text {max }}-S_{\text {min }}\right)$

In the evaluation of the ICP when using priority matrix, distance matrix, the number of bad indicators matrix as a comparison scale.

$$
\begin{aligned}
& (c m(k, j))_{n_{c} \times m_{a}}=\sum_{i \in p c(k, j)} w_{i} / \sum_{i=1}^{n_{c}} w_{i} \\
& p c(k, j)=\left\{c_{i} \mid\left(a_{k} \succ a_{j}\right) \cup\left(a_{k} \sim a_{j}\right)\right\}
\end{aligned}
$$

In learning who whole learning process in the emotional for analysis can informed its personalized of learning information, and will its data for different dimension degrees of classification, to provide personalized of learning support $\$$ which, needed reference "fuzzy number" of concept, because learning who individual in learning process in the of cognitive State, and learning preference, and knowledge based of uncertainty can through on target learning object of cognitive reaction and emotional attitude for data poly class, to on its for overall assessment and management. For example, understanding the learners to the concept of a learning effect, can be inferred from its historical cognition on cognition of this concept (learning object has different difficulty levels), but because of the learner's personality cognition with dynamic uncertainty and be determined upon completion of the concept learning in learners to further their understanding of, and to gradually reduce cognitive fuzzy numbers. Through long-term accumulation, not only to the learner's cognitive state accurately, and can provide appropriate feedback to the learner learning content.

\section{RESULTS}

At the early stage on the basis of a series of tests, analytical work, provide effective feedback to the learner is finally part of emotion detection is the ultimate goal. This a technology steps is through on education agent and learning whose interactive information of early detection, completed on learning who of learning monitoring, to provides further of learning support, including learning strategy and personalized learning path of feedback $\$$ due to each courses of learning are has its specific of learning target and content, and to for learning who provides personalized of courses learning 


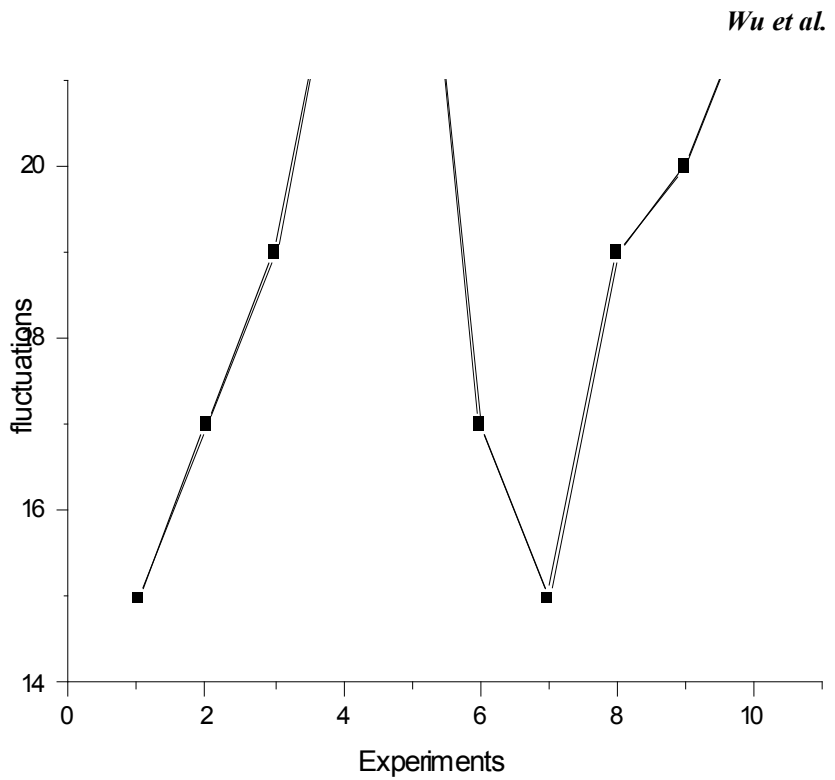

Fig. (1). Human-computer interaction in the process of distance learners' emotional effect.

process, automatically feedback function of design is important. Different from the present goal of classroom learning, automatic generation of the learning objectives of the course is based on the learners ' personality information. Although has established of total courses target, but in learning process in the of unit learning target is undecided of, is constantly dynamic update changes of, has sharp of personality features and dynamic sex $\$$ in whole learning process in the, by education agent served as of "Mentor" not by text of limit, but through and learning who carried out interactive, in interactive in the generated new courses, and courses itself also no longer is still, and fixed, and linear to rendering knowledge content, but with learning who of learning State, and emotional attitude changes constantly dynamic generated, According to the level in the learning process and constantly progressive with, which went beyond the original architecture of the course, belongs to the learner's "private custom" course. Automatically generate curriculum provided by the different learning contexts and appropriate learning strategies become available to the learner's self beyond the driving force to make it in the learning process are no longer content to established learning objectives of the course of the acquisition of knowledge and skills, but pay more attention to activities of the inquiry learning of complex situations, and with existing known The organic integration of knowledge, experience, and creatively to construct their own knowledge and experience system.

Metacognition monitoring includes cognitive and learning preferences and knowledge three factors, cognitive state is for a particular test knowledge, conceptual level of cognition and memory. For example, learners of different cognitive status, may be on the same target learning, the study time and master degrees are different, so the sequence of learning objects to be designed to accommodate learners' personality; learning preference refers to the learner's perception that learners learn what objects are more likely to accept or be attracted. Assessment of students ' preferences,
Fig. (2). Detection distance learners' affective interactive process fluctuations.

are using different metadata category inclusion in the metadata element is extracted. Such as media, teaching semantic density and degree of interaction, and so on; Knowledge refers to the knowledge gained in previous learning experiences, including in this learning process available knowledge and learning other than the accumulation of knowledge. For remote-Learner's affective information portfolio, there are three categories of users: learners, teachers, and administrators. Emotional information archives bags for each learning who provides records emotional changes, and emotional exchange, and emotional evaluation of space, and gives teachers and students browse and evaluation learning who emotional of right; while, emotional information archives bags also requirements teachers developed emotional target and according to emotional target on learning who emotional for corresponding of evaluation and adjustment; administrator is archives bags of managers, its main task is archives bags resources management and system maintenance.

Affective model is actually stored as database the learner's emotional state, and its main function is to manage archives determination of learners and learners ' emotional state. Emotional information management capabilities including learning to create, modify, and delete. (1) $\operatorname{login} /$ register. When learners $\log$ on emotion when the information portfolio, testing whether the learner has registered emotion model, if not, register and $\log$ in; or directly login and give their number and login information such as frequency and duration. (2) The emotion before learning tests. Responsible for testing learners' learning styles, study habits, attitude, interest, before the learner's emotional information. (3) The study of emotion in the record. Is the main parts of model of emotion, the main emotion is in the class change, interactions with others, and log on to the system frequency and emotional information to affective information such as progress portfolio. (4) The emotions after learning summary. After the study is over, record response to learners ' learning feelings summarize and emotional control 


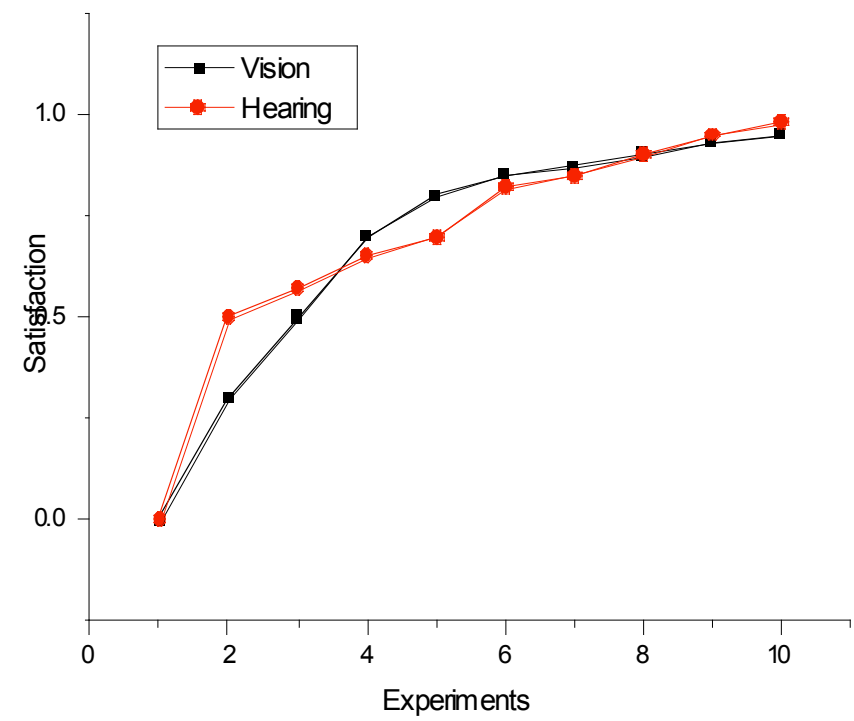

Fig. (3). Human-computer interaction in the process of distance learners' emotional index difference.

of State information. (5) Evaluation and reflection on the information. Throughout the learning process of selfreflection, for the learner and others the affective on the learner's evaluation information to emotional information are in the portfolio. (6) Emotional information. Display various emotions reflect learner status information. (7) A search query. Providing learners search for key words or concepts so that students can have a better understanding of their learning. (8) Emotion regulation. According to the learner's learning, evaluation and reflection on affective and good learning, emotional compensation are provided by the teacher or learner emotional adjustment. (9) The emotional information maintenance module. Responsible for editing and management of emotion model in emotion and so on.

\section{CONCLUSION}

Emotion detection to distance learners is a highly integrated technology, through psychology and cognitive sciences, sociology, biology, and other disciplines of a harmonious fusion, completed and education agents, the interaction between the learner and the display terminal activities, and technical feedback, According to the learner's emotional changes to provide additional learning support $[8,9]$. This will undoubtedly improve the interactive means, not only to upgrade and expand distance learning under the background of cloud computing to create a technology space, while also learning to the learner and emotional information capture and subsequent learning strategy provides strong support for further feedback [10]. But due to technical constraints, emotion detection application still faces the following challenges: (1) emotion corpus establishment and expansion. Sources of information such as corpus selection, filtering of input information and of corpus structure refinement; (2) affective information access. This study was based on emotional voice and text detection, transcription for speech signals, accuracy during transcription sure there have been problems. Coupled with emotional has strong of social and culture sex, voice signal and text information itself has complexity, these factors are makes on teach who emotional information of gets faced heavy difficult; (3) education agent of language feedback. In learning process, learning and education agent take dialogue form for communication and interactive, for agent expression content of precision degrees by voice signal and situation of effect. But even so, emotion detection distance learners still have a wide range of applications, in order to effectively address human-computer interaction in distance learning issues and improve learning efficiency provide excellent technical support [11-13].

We know of no previous register-based study that has illustrated the relevance of these two crucial issues in an equally detailed manner as we have done here.

\section{CONFLICT OF INTEREST}

The authors confirm that this article content has no conflict of interest.

\section{ACKNOWLEDGEMENTS}

This work is supported by the National Natural Science Foundation of China (51275447); the Science and Technology Support Program of Jiangsu (BE2011133); the Natural Science Foundation of Jiangsu (13KJB460015)

\section{REFERENCES}

[1] Liu, Jing, J Tong, J Han, S.Chen, and F Yang, "Affective Computing Applications in Distance Education," In: The International Conference on Education Technology and Information System (ICETIS 2013), pp. 927-930, 2013.

[2] L. Shen, V. Callaghan, and R. Shen, "Affective e-Learning in residential and pervasive computing environments," Information Systems Frontiers, vol. 10, no. 4, pp. 461-472, 2008.

[3] I. S. MacKenzie, "Fitts' law as a research and design tool in humancomputer interaction," Human-computer interaction, vol. 7, no. 1, pp. 91-139, 1992.

[4] D. E. Kieras, and D. E. Meyer, "An overview of the EPIC architecture for cognition and performance with application to humancomputer interaction," Human-computer interaction, vol. 12, no. 4, pp. 391-438, 1997.

[5] S. Brave, and C. Nass, "Emotion in human-computer interaction," Human-Computer Interaction, vol. 53, pp. 81-96, 2003.

[6] S. L. Rossell, T.E. Van Rheenen, C. Groot, A. Gogos, A. O'Regan, and N.R. Joshua, "Investigating affective prosody in psychosis: a study using the comprehensive affective testing system," Psychiatry Research, vol. 210, no. 3, pp. 896-900, 2013.

[7] J. J. Hedl, H. F. O'Neil, and D. N. Hansen, "Affective reactions toward computer-based intelligence testing," Journal of Consulting and Clinical Psychology, vol. 40, no. 2, pp. 217-222, 1973.

[8] J. Schuessler, and P. Burger, "Reaching the Remote Learner through Web-Conferencing: A Testimonial on using Collaboration, Self, and Peer-Assessment to Turn the Focus from Grades to Learning," Global Time, vol. 12, no. 1, pp. 6, 2012.

[9] M. Pantic, and J.M. R. Leon, "Toward an affect-sensitive multimodal human-computer interaction," In: Proceedings of the IEEE, vol. 91, no. 9, pp. 1370-1390, 2003.

[10] Pantic, Maja, Nicu Sebe, F. Jeffrey, and T. Huang, "Affective multimodal human-computer interaction," In: Proceedings of the 13th annual ACM international conference on Multimedia. ACM, pp. 669-676, 2005.

[11] . Timo, and V. Surakka, "The effects of affective interventions in human-computer interaction," Interacting with computers, vol. 16, no. 2, pp. 295-309, 2004. 
[12] J. Morkes, K. K. Hadyn, and C. Nass, "Effects of humor in taskoriented human-computer interaction and computer-mediated communication: A direct test of SRCT theory," Human-Computer Interaction, vol. 14, no. 4, pp. 395-435, 1999.
[13] Y. Rogers, S. Helen, and P. Jenny, "Interaction design: beyond human-computer interaction," John Wiley \& Sons, 2011.

Received: June 10, 2015

Revised: July 29, 2015

Accepted: August 15,2015

(C) Wu et al.; Licensee Bentham Open.

This is an open access article licensed under the terms of the (https://creativecommons.org/licenses/by/4.0/legalcode), which permits unrestricted, noncommercial use, distribution and reproduction in any medium, provided the work is properly cited. 